\title{
Cardiovascular and Metabolic Health of 74 Children From Women Previously Diagnosed With Polycystic Ovary Syndrome in Comparison With a Population-Based Reference Cohort
}

Reproductive Sciences

2018, Vol. 25(10) 1492-1500

(C) The Author(s) 2018

Article reuse guidelines:

sagepub.com/journals-permissions DOI: $10.1|77 /| 9337|9| 1774976$

journals.sagepub.com/home/rsx

@SAGE

\author{
Marlieke A. de Wilde, MD', Jacobien B. Eising, MD, PhD², \\ Marlise N. Gunning, MD, MSc', Maria P. H. Koster, MD, PhD', \\ Annemieke M. V. Evelein, MD, PhD $^{3}$, Geertje W. Dalmeijer, PhD $^{3}$, \\ Cuno S. P. M. Uiterwaal, MD, PhD ${ }^{3}$, Marinus J. C. Eijkemans, PhD ${ }^{1,3}$, \\ Cornelis K. van der Ent, MD, PhD ${ }^{2}$, Folkert J. Meijboom, MD, PhD ${ }^{4}$, \\ and Bart C. J. M. Fauser, MD, PhD $^{1}$
}

\begin{abstract}
Women with polycystic ovary syndrome (PCOS) have compromised cardiovascular health profiles and an increased risk of pregnancy complications. In order to evaluate potential consequences, we aim to compare the cardiovascular and metabolic health of the children from women with PCOS with a population-based reference cohort. We included children from women with PCOS between the age of 2.5 to 4 years $(n=42)$ and 6 to 8 years $(n=32)$. The reference groups consisted of $168(3-4$ years old) and 130 children (7-8 years old). In an extensive cardiovascular screening program, we measured anthropometrics and blood pressure (all children), heart function and vascular rigidity (young children), metabolic laboratory assessment and carotid intima thickness (old age-group). Results showed that young PCOS offspring have a significantly lower diastolic blood pressure ( $\beta=2.3$ [95\% confidence interval, $\mathrm{Cl}: 0.5-4.0])$ and higher aortic pulse pressure $(\beta=-\mathrm{I} .4$ [95\% $\mathrm{Cl}:-2.5$ to -0.2$])$, compared to the reference population. Furthermore, a higher left ventricle internal diameter but a lower tissue Doppler imaging of the right wall in systole compared to the reference group was found. Older offspring of women with PCOS presented with a significantly lower breast and abdominal circumference, but higher triglycerides $(\beta=-0.1$ [95\% Cl: -0.2 to -0.1$])$, LDL-cholesterol $(\beta=-0.4$ [95\% $\mathrm{Cl}:-0.6$ to $-0 . \mathrm{I}])$, and higher carotid intima-media thickness $(\beta=-31.7[95 \% \mathrm{Cl}:-46.6$ to -16.9$])$ compared to the reference group. In conclusion, we observe subtle but distinct cardiovascular and metabolic abnormalities already at an early age in PCOS offspring compared to a population-based reference group, despite a lower diastolic blood pressure, breast, and abdominal circumference. These preliminary findings require confirmation in independent data sets.
\end{abstract}

\section{Keywords}

cardiovascular, metabolic, offspring, PCOS

\section{Introduction}

Polycystic ovary syndrome (PCOS) is a common endocrine disorder, affecting $6 \%$ to $15 \%$ of all women of reproductive age. ${ }^{1,2}$ According to the Rotterdam consensus criteria, PCOS is diagnosed when at least 2 of the following 3 criteria are present (after exclusion of other potential causes): oligo- or anovulation, polycystic ovaries, and clinical or biochemical signs of hyperandrogenism. ${ }^{3}$ Other features such as infertility, obesity, and metabolic dysfunction (eg, insulin resistance, dyslipidemia) often coincide in women with $\mathrm{PCOS} .^{2}$ Familial clustering of PCOS suggests a heritable component of this heterogeneous condition, although the genes involved remain elusive. ${ }^{4-6}$

\footnotetext{
' Department of Reproductive Medicine and Gynaecology, University Medical Center Utrecht, Utrecht University, Utrecht, The Netherlands

2 Department of Paediatric Pulmonology, University Medical Center Utrecht, Utrecht University, Utrecht, The Netherlands

3 Julius Centre for Health Sciences and Primary Care, University Medical Center Utrecht, Utrecht University, Utrecht, The Netherlands

${ }^{4}$ Department of Paediatric Cardiology, Wilhelmina Children's Hospital, University Medical Center Utrecht, Utrecht University, Utrecht, The Netherlands
}

\section{Corresponding Author:}

Marlise N. Gunning, Department of Reproductive Medicine and Gynaecology, University Medical Center Utrecht, Heidelberglaan 100, 3584 CX Utrecht, The Netherlands.

Email: m.n.gunning@umcutrecht.nl 
Besides a presumed genetic susceptibility, other features associated with PCOS may affect future health of offspring, such as obesity and unfavorable metabolic dysfunction of the mother, ${ }^{7}$ placental abnormalities, and increased pregnancy complications. ${ }^{8,9}$ The concept - initially put forward by Barkerthat periconceptional health of women along with subsequent embryo development during early pregnancy affects the future health of offspring is increasingly recognized. ${ }^{10}$

It has been demonstrated that PCOS offspring may have an increased risk of hyperinsulinemia and insulin resistance from puberty onward. ${ }^{11-13}$ Insulin resistance is associated with endothelial dysfunction, which may emerge already during childhood by accumulation of lipids within the blood vessel walls. ${ }^{14,15}$ As a result, children from women with PCOS might also show signs of vascular changes early in life. However, a single study in 76 PCOS daughters observed no difference in glucose-challenged salivary insulin concentrations compared to controls. ${ }^{16}$

Potential cardiovascular and metabolic implications for offspring of well-phenotyped women with PCOS have not been studied in great detail. According to a single study, eumenorrheic nonhirsute young adult daughters with a mean age of 24 years of women with PCOS present with unfavorable cardiovascular features such as an increased blood pressure and increased fasting insulin when compared to age and body mass index (BMI) matched controls. ${ }^{17}$ Other studies in PCOS offspring mainly focused on reproductive features and metabolic consequences of PCOS during childhood. ${ }^{11-13,18}$ Moreover, potential sex differences in PCOS offspring remain poorly studied.

We hypothesize that the children from women with PCOS may already have subtle signs of reduced metabolic and cardiovascular health early in life. Therefore, the aim of the current study was to compare cardiovascular and metabolic health in children from women with PCOS at 2.5 to 4 years and 6 to 8 years of age, with children of comparable age from a population-based reference group.

\section{Methods}

Design

Children of women with PCOS. Children were recruited by women who were approaching extensively screened and diagnosed with PCOS at the outpatient clinic of the department of reproductive medicine of the University Medical Centre Utrecht (UMCU) from 2004 onward. ${ }^{3,19}$ Women with PCOS who delivered a live born baby between January 2005 and December 2010 were asked to participate with their child in The CHildren follow-up Of women with PcoS (CHOPS) study. Exclusion criteria for children participating in our study were heart defect, type I diabetes, or a respiratory infection less than 2 weeks before screening. Written informed consent was obtained from both parents, and screening took place between February 2013 and July 2014.

Reference group. The design of the CHOPS study deliberately has great similarities with the reference cohort to improve comparability between study populations. The reference group was also recruited from the similar region of Utrecht, the Netherlands, and was part of the WHeezing Illnesses STudy LEidsche Rijn (WHISTLER). All newborns born since 2001 in Leidsche Rijn near the city of Utrecht were invited for this population-based follow-up study. Exclusion criteria were gestational age $<36$ weeks, major congenital abnormalities, or neonatal respiratory disease. The study design and rationale of the parental follow-up study and the offspring study WHISTLER and WHISTLER-cardio were previously published elsewhere. ${ }^{20-22}$

Funding bodies. The Child Health research program of the UMCU, the Dutch Heart Foundation (2013T083), ZonMw (2100.0095), and GlaxoSmithKline, had no role in the design, data collection, analysis and interpretation of data, or writing of the article, or the decision to submit the article for publication.

\section{Questionnaires and Clinical Assessments}

Differences between the 2 age groups occurred, the widely used method for assessing cardiovascular risk, a carotid intima-media thickness measurement (IMT) ${ }^{23}$ is impossible in the youngest group due to a discrepancy between the size of the ultrasound transducer and the neck dimensions. Furthermore, due to patient involvement in the design of this study, we refrained from blood withdrawal within the younger age-group.

Both age categories. Before screening, a questionnaire was sent to the parents, gathering information on health status of both parents, family history of cardiovascular diseases and pregnancy information (gestational diabetes mellitus [GDM], pregnancy-induced hypertension [PIH], preeclampsia [PE], and the hemolysis, elevated liver enzymes, and low platelets syndrome). Data on menstrual cycle pattern, mode of conception, and parity were unavailable among mothers from the WHISTLER cohort. ${ }^{21,24}$

Screening in younger study group. Weight and height of the child were measured while being bare foot and in underclothing with a standard electronic scale and a stadiometer, respectively. A nonelastic tape measure was used for circumference measurements. Breast circumference was measured twice after expiration at the height of the nipples. Waist circumference was assessed twice after expiration between the superior iliac anterior spine and the lowest rib. Furthermore, blood pressure and vascular rigidity were measured using arteriography. A transthoracic echocardiogram was also performed using the Vivid 7 ultrasound machine (GE Healthcare, Milwaukee and Wauwatosa, Wisconsin) with a $6 \mathrm{~S}, 2.7$ to $8.0 \mathrm{MHz}$ transducer with continuous electrocardiographic monitoring. ${ }^{21,24}$ All echocardiograms were performed by the same medical doctor (J.B.E.) who was trained intensively. Echocardiographic measurements were performed according to the guidelines of the pediatric council of the American Society of Echocardiography ${ }^{25,26}$ and were previously described elsewhere. ${ }^{27}$ Analyses of the 
echocardiograms of both study groups were performed by 1 observer (M.A.W.) who was blinded for clinical measurements of the children and was supervised by an experienced cardiologist (F.J.M.).

Older study group. Anthropometrics and blood pressure were measured similar to the children of the younger age category. Subsequently, a carotid IMT measurement (in supine position) was performed. ${ }^{21,24}$ The carotid IMT of the far wall and the diameter during diastole and the distension (change in diameter from diastole to systole) of the right common artery were assessed by using high resolution echo-tracking technology (Art.lab, Esaote, Genoa, Italy) including a 128 line radiofrequency line multiarray, with an L10-5 $40 \mathrm{~mm}$ linear array transducer. ${ }^{28}$ The whole procedure was described elsewhere. ${ }^{22}$ Averages of the distension, lumen, and the carotid IMT were used to demonstrate the arterial elasticity by cross-sectional distensibility and the elastic modulus. These measurements were performed by a medical doctor (A.M.V.E.).

\section{Laboratory Assessments}

A blood sample was asked from all children in the older study groups, which was processed and stored at $-80^{\circ} \mathrm{C}$, immediately after withdrawal. In the reference group, fasting glucose, fasting insulin, total cholesterol, triglycerides, HDL-cholesterol and $\mathrm{C}$-reactive protein (CRP) were determined immediately after withdrawal. In the PCOS offspring group, assessments were performed after storage in a $-80^{\circ}$ freezer. The analyses within both study groups were previously used and performed with a DxC800/AU5811 (Beckman Coulter, Fullerton, California), and fasting insulin was measured on the Modular E170 (Roche Diagnostics, Mannheim, Germany). ${ }^{29}$ A homeostatic model assessment insulin resistance (HOMA-IR) was calculated as a measure of insulin resistance with the following formula: [HOMA-IR $=($ glucose $\times$ insulin $) / 22.5]$. LDL-cholesterol was calculated with the formula of Friedewald.

\section{Statistical Analysis}

The sample size was based on children who were conceived by assisted reproductive technologies. Their pulse wave velocity was significantly higher (mean 7.8 [2.4]; a standardized difference [Cohen $D]=0.5$, assuming the largest of the 2 standard deviations). ${ }^{30}$ The study was designed to include offspring from women with PCOS and reference participants in a ratio of 1:4. A further increase of the reference group has no additional value. ${ }^{31}$ A statistical power of $80 \%$ would be achieved to detect a standardized difference of 0.5 , when 40 PCOS children were included per age category. In case 30 PCOS children were included, power for this difference would still be close to $70 \%$. All data were analyzed using SPSS Statistics (IBM SPSS Inc, Chicago, Illinois, version 21.0). A BMI was calculated for both groups: [Weight/Length $\left.{ }^{2}\right]$. Means and standard deviations were calculated for all normally distributed continuous variables and medians with interquartile ranges for all nonnormally distributed variables. Missing data were handled by using single imputation.

The multivariate analysis of variance (MANOVA) test was used on different composite outcomes, of which each composite outcome comprised a different domain of offspring health to refrain from multiple testing. In case the overall test was significant, we used the MANOVA post hoc test. The generalized linear models function in SPSS was used for MANOVA. Age- and sex-specific BMI $Z$ scores were calculated based on data of Dutch children from the Dutch organization for applied scientific research (TNO). In multivariable analyses, we adjusted for age- and sex-adjusted BMI $Z$ scores and multiple gestations. We decided to adjust for sex in a sensitivity analysis since the effect of sex on cardiovascular and metabolic health is not clear yet in young children (see Supplemental Material). We refrained from a matched study design since this does not control for confounding by the matching factors. It could even introduce confounding by the matching factors. ${ }^{32,33}$ Data on maternal and pregnancy characteristics were analyzed by using univariate analyses: Student $t$ tests (normally distributed data) and Wilcoxon rank sum test (nonparametric data). And for nominal and ordinal $\chi^{2}$ test or Fisher exact test (in case of little events were present). $P$ values $<.05$ were considered statistically significant. $Z$ Scores were determined for the diameter of the left end-diastolic and end-systolic internal ventricle based on a study in 2036 children at the age between 1 month and 18 years in Central Europe. ${ }^{34}$

\section{Results}

Hundred and thirteen women with PCOS had children from 2.5 to 4 or 6 to 8 years old. A few women declined participation because of the travel time $(n=19)$ or the burden for their child $(n=18)$. One child was excluded because of a type I diabetes mellitus, and parents of 2 children did not provide a reason. A total of 42 children (2.5-4 years) and 32 children (6-8 years) were included (CHOPS). The reference groups consisted of 168 children (3-4 years) and 130 children (7-8 years; WHISTLER). Tables 1 and 2 show that the age of the CHOPS groups in both age categories was significantly lower than the reference groups (3.3 [0.6] vs 3.7 [0.2] and 7.0 [0.8] vs 8.1 [0.5], respectively, both $P<.001$ ).

\section{Young Study Group (Between 2.5 and 4 Years)}

The MANOVA post hoc test shows that the diastolic blood pressure is significantly lower in PCOS offspring $(\beta=2.3$ [95\% confidence interval, CI: $0.5-4.0], P=.012$; Table 1). The MANOVA post hoc test on arterial stiffness shows that the pulse pressure was significantly higher in PCOS offspring ( $\beta=-1.4$ [95\% CI: -2.5 to -0.2$], P=.021$ ). The echocardiographic measurements in the young study group were available for $86 \%(\mathrm{n}=36)$ of all children in the PCOS group and $81 \%$ $(n=136)$ of all children in the reference group. No statistically significant were found between children of available and unavailable echocardiography data (data not shown). Children in the 
Table I. Anthropometrics, Blood Pressure, Arteriographics, and Echocardiographics (Means and SD) in 42 PCOS Children and I68 Children in the Reference Group (Age Range: 2.5-4 Years). ${ }^{\mathrm{a}}$

\begin{tabular}{lccc}
\hline Characteristics & $\begin{array}{c}\text { CHOPS PCOS } \\
\text { Children } \\
(\mathrm{n}=42)\end{array}$ & $\begin{array}{c}\text { WHISTLER } \\
\text { Reference Group } \\
(\mathrm{n}=168)\end{array}$ & $\begin{array}{c}\text { Linear Regression: } \\
P \text { Value }\end{array}$ \\
\hline Age, Years & $3.3(0.6)$ & $3.7(0.2)$ & $<.001$ \\
Height, cm & $100.2(5.5)$ & $102.2(4.5)$ & $.892^{\mathrm{b}}$ \\
Weight, kg & $16.1(2.6)$ & $17.1(2.3)$ & $.652^{\mathrm{b}}$ \\
BMI, kg/m & $15.9(1.6)$ & $16.4(1.3)$ & $.293^{\mathrm{b}}$ \\
\hline
\end{tabular}

MANOVA With Post Hoc Test': $P$ Value Adjusted for SexAge-Specific-BMI Z score ${ }^{d}$ and Multiple Gestation

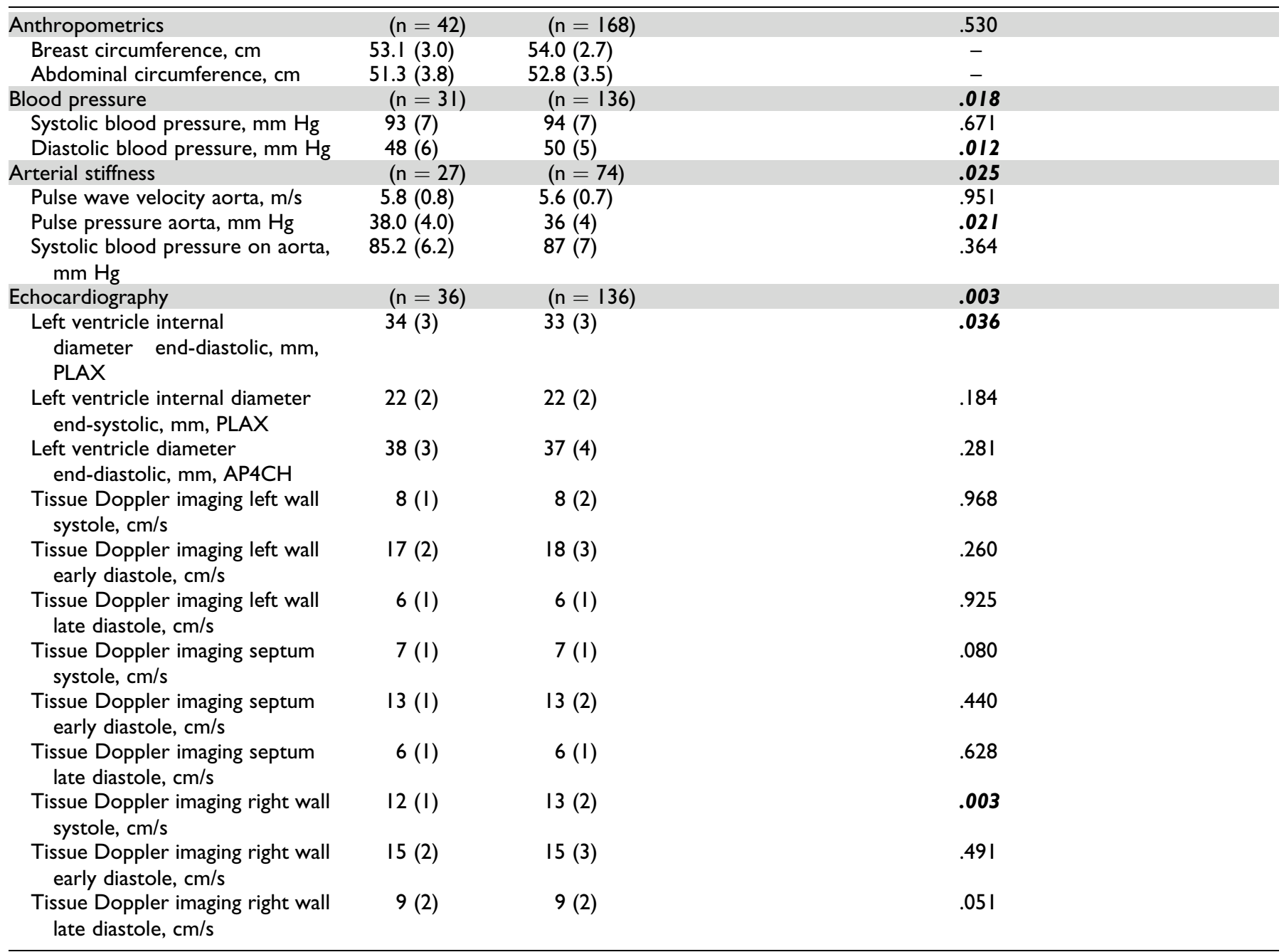

Abbreviations: AP4CH, apical four chamber; BMI, body mass index; CHOPS, The CHildren follow-up Of women with PcoS; $\mathrm{cm}$, centimeter; mm Hg, millimeter of mercury; n, number; PCOS, polycystic ovary syndrome; PLAX, parasternal long axis; WHISTLER, WHeezing Illnesses STudy LEidsche Rijn; MANOVA, multivariate analysis of variance.

${ }^{\mathrm{a}}$ Italics and boldface indicates statistical significance at $P<.05$.

'Linear regression adjusted for age.

'MANOVA (overall $P$ value in gray row) with post hoc test: MANOVA with a post hoc test for which the results from the post hoc test will only be displayed if the overall MANOVA was significant.

${ }^{\mathrm{d}}$ Age- and sex-specific BMI $Z$ score based on the Dutch population.

PCOS group did show significant differences in the following 2 parameters: a higher end-diastolic left ventricle internal diameter $(\beta=-0.986$ [95\% CI: -1.909 to -0.063$], P=.036)$ and a lower systolic tissue Doppler imaging of the right wall $(\beta=0.9$
[95\% CI: 0.3-1.5], $P=.003$ ) compared to the reference group. Additionally, adjusting for sex shows similar significant findings in almost every, except for one borderline significant and one borderline nonsignificant, outcome (see Supplemental Material). 
Table 2. Anthropometrics, Blood Pressure, and Carotid Intima Media Thickness (Means and SD) for 32 PCOS Children and I 30 Children in the Reference Group (Age Range: 6-8 Years). ${ }^{a}$

\begin{tabular}{lccc}
\hline & $\begin{array}{c}\text { CHOPS } \\
\text { PCOS Group } \\
(\mathrm{n}=32)\end{array}$ & $\begin{array}{c}\text { WHISTLER } \\
\text { Reference Group } \\
(\mathrm{n}=130)\end{array}$ & $\begin{array}{c}\text { Linear Regression } \\
P \text { Value }\end{array}$ \\
\hline Age, years & $7.0(0.8)$ & $8.1(0.5)$ & $<.001$ \\
Weight, kg & $23.8(4.3)$ & $27.9(4.2)$ & $.439^{\mathrm{b}}$ \\
Height, $\mathrm{cm}$ & $125(7)$ & $132(5)$ & $.050^{\mathrm{b}}$ \\
BMI, kg/m & $15.3(1.5)$ & $15.8(1.7)$ & $.841^{\mathrm{b}}$ \\
\hline
\end{tabular}

MANOVA With Post Hoc Test ${ }^{c}: P$ Value Adjusted for Age-Specific-BMI z Score and Multiple Gestation ${ }^{\text {d }}$

\begin{tabular}{|c|c|c|c|}
\hline \multicolumn{3}{|l|}{ Anthropometrics } & \multirow{2}{*}{$\begin{array}{l}<.001 \\
<.001\end{array}$} \\
\hline Breast circumference, $\mathrm{cm}$ & $58.6(4.0)$ & $62.6(4.1)$ & \\
\hline Blood pressure & $(n=32)$ & $(n=129)$ & .528 \\
\hline Diastolic blood pressure, $\mathrm{mm} \mathrm{H} \mathrm{H}$ & $55(6)$ & $54(6)$ & - \\
\hline Blood assessments & $(n=14)$ & $(n=130)$ & $.04 I$ \\
\hline Glucose, mmol/L & $4.7(0.4)$ & $4.6(0.3)$ & .632 \\
\hline Cholesterol, $\mathrm{mmol} / \mathrm{L}$ & $4.9(1.3)$ & $4.4(0.7)$ & .134 \\
\hline Triglycerides, $\mathrm{mmol} / \mathrm{L}$ & $0.7(0.2)$ & $0.5(0.2)$ & .031 \\
\hline HDL-cholesterol, $\mathrm{mmol} / \mathrm{L}$ & I.5 (0.3) & $1.4(0.2)$ & .476 \\
\hline LDL-cholesterol, $\mathrm{mmol} / \mathrm{L}$ & $3.1(1.3)$ & $2.8(0.6)$ & .015 \\
\hline $\mathrm{CRP}, \mathrm{mg} / \mathrm{L}$ & $1.6(4.0)$ & $2.5(3.1)$ & .371 \\
\hline Carotid intima media thickness & $(n=32)$ & $(n=130)$ & .001 \\
\hline
\end{tabular}

Abbreviations: BMI, body mass index; CHOPS, The CHildren follow-up Of women with PcoS; CRP, C-reactive proteine; cm, centimeter; HOMA-IR, homeostasis model assessment-estimated insulin resistance; $\mathrm{kPa}$, kilo Pascal; $\mathrm{mlU} / \mathrm{L}$, Milli-International Units Per Liter; $\mathrm{mm} \mathrm{Hg}$, millimeter of mercury; mmol/L, millimoles per liter; $\mu \mathrm{m}$, micrometer; $\mathrm{n}$, number; PCOS, polycystic ovary syndrome; WHISTLER, WHeezing Illnesses STudy LEidsche Rijn.

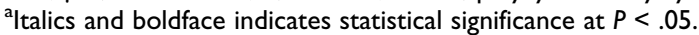

binear regression adjusted for age.

'MANOVA (overall $P$ value in gray row) with post hoc test: MANOVA with a post hoc test of which the results from the post hoc test will only be displayed if the overall MANOVA was significant.

${ }^{\mathrm{d}}$ Age- and sex-specific BMI Z score based on the DUTCH population.

eHOMA-IR was not included in the MANOVA for the 2 components of HOMA-IR (glucose, insulin) were already taken into account separately in the MANOVA.

\section{Older Study Group (Between 6 and 8 Years)}

After adjusting for confounders, lower breast and abdominal circumferences were found in the PCOS offspring compared to the reference group ( $\beta=3.2$ [95\% CI: 2.3-4.2], $P<.001$ and $\beta$ $=2.8$ [95\% CI: 1.7-3.8], $P<.001$, respectively). Unlike in the WHISTLER cohort, blood assessments could only be performed in 14 (44\%) children. The CHOPS children from whom blood samples were withdrawn did not differ significantly (see Supplemental Material). Triglycerides ( $\beta=-0.1$ [95\% CI: -0.2 to -0.1$], P=.031)$ and LDL-cholesterol $(\beta=-0.4$ [95\% CI: -0.6 to -0.1 ] $P=.015$ ) were significantly higher in the PCOS group in comparison with the reference group. Furthermore, the carotid IMT was significantly higher compared to the reference group ( $\beta=-31.7$ [95\% CI: -46.6 to -16.9 ], $P<.001)$. After additional adjustment for sex, all significant outcomes remained the same (see Supplemental Material).

\section{Pregnancy and Birth Characteristics of PCOS Children Versus Reference Group}

Mothers with PCOS differed significantly in mode of delivery compared to the reference group $(P=.007$; Table 3$)$. In $27 \%$ ( $\mathrm{n}=$ $20)$ of the PCOS mothers, at least 1 pregnancy complications is present, which is only $11 \%(\mathrm{n}=33)$ in the reference group $(P=$ $0.001)$. Gestational diabetes is prevalent in $14 \%(\mathrm{n}=10)$ in PCOS mothers and in $1.7 \%(\mathrm{n}=5)$ in the reference group $(P<.001)$. No difference was observed in occurrence of children born small or large for gestational between the CHOPS and WHISTLER cohorts. Birth weight as a continuous outcome did show significant differences with a lower birth weight in PCOS offspring $(P=$ $.005)$. Mode of conception in women with PCOS was spontaneous in $31 \%(n=27)$, ovulation induction in $45 \%(n=33)$ using clomiphene citrate $27 \%(\mathrm{n}=20)$ and gonadotrophins in $18 \%$ $(\mathrm{n}=13)$. In vitro fertilisation (IVF) or intracytoplasmic sperm injection (ICSI) was performed in $12 \%$ of all pregnancies $(n=9)$. 
Table 3. Maternal, Pregnancy, and Birth Characteristics of the Entire Study Population (Means and SD or Number of Occurrence and Percentages). ${ }^{\mathrm{a}}$

\begin{tabular}{|c|c|c|c|}
\hline Characteristics & $\begin{array}{l}\text { CHOPS PCOS Children } \\
\qquad(\mathrm{n}=74)\end{array}$ & $\begin{array}{l}\text { WHISTLER Reference Group } \\
\qquad(\mathrm{n}=298)\end{array}$ & $\begin{array}{l}\text { Univariate Analysis } \\
\qquad \text { Value }^{\mathrm{b}}\end{array}$ \\
\hline \multicolumn{4}{|l|}{ PCOS criteria during preconception } \\
\hline Biochemical hyperandrogenism $(\mathrm{FAl}>4.5)$ & $40(54.1 \%)$ & NR & \\
\hline Polycystic ovaries on ultrasound & $64(86.5 \%)$ & NR & \\
\hline Maternal BMI at visit $\left(\mathrm{kg} / \mathrm{m}^{2}\right)$ & $25.6(5.7)$ & $24.5(4.0)$ & $.405^{\mathrm{b}}$ \\
\hline Any maternal complication & $20(27.0 \%)$ & $33(11.1 \%)$ & $.001^{d}$ \\
\hline Gestational diabetes & $10(13.5 \%)$ & $5(1.7 \%)$ & $<.00 I^{d}$ \\
\hline Any hypertensive complication & $9(12.2 \%)$ & $28(9.7 \%)$ & $.523^{d}$ \\
\hline Gestational age at delivery & $39.1(2.6)$ & $39.8(1.4)$ & $.157^{b}$ \\
\hline Multiple gestation & $4(5 \%)$ & $8(3 \%)$ & $.266^{\mathrm{d}}$ \\
\hline Mode of delivery & & & $.007^{\mathrm{e}}$ \\
\hline Newborn sex: male & $38(5 \mathrm{I} .4 \%)$ & $125(42.2 \%)$ & $.190^{d}$ \\
\hline Birth weight, gr, mean and SD & $3.304(644)$ & $3.569(487)$ & $.005^{\mathrm{b}}$ \\
\hline \multicolumn{4}{|l|}{ Neonatal complications } \\
\hline Small for gestational age & $7(9.5 \%)$ & $6(3.6 \%)$ & $.069^{\mathrm{d}}$ \\
\hline Large for gestational age & $6(8.1 \%)$ & $26(15.5 \%)$ & $.151^{d}$ \\
\hline
\end{tabular}

Abbreviations: BMI, body mass index; CHOPS, The CHildren follow-up Of women with PcoS; FAl, free androgen index; HELLP, hemolysis, elevated liver enzymes, low platelets; kg, kilogram; kg/m, kilogram per square meter; n, number; NR, not reported; PCOS, polycystic ovary syndrome; WHISTLER, WHeezing IIlnesses STudy LEidsche Rijn.

${ }^{a}$ Italic and boldface indicates statistical significant with $P<.05$.

bUnivariate analyses performed with Mann-Whitney $U$ test.

'Some women presented with 2 pregnancy complications.

dUnivariate analyses performed with Fishers exact test.

eUnivariate analyses performed with $\chi^{2}$-test.

\section{Discussion}

\section{Main Findings}

In the current study, we observed subtle but distinct abnormalities in both cardiovascular and metabolic features in PCOS children, already at an early age. These differences are reflected by an increased pulse pressure and a higher left ventricular internal diameter (end-diastolic) but a lower tissue Doppler imaging of the right wall in systole at 2.5 to 4 years of age. At the age of 6 to 8 years, we observed a higher carotid IMT and higher serum levels of total triglycerides and LDL-cholesterol in children of women previously diagnosed with PCOS, regardless of a lower breast and abdominal circumference.

In the younger children, we observed a higher pulse pressure within the CHOPS cohort. Pulse pressure rises with increasing arterial stiffness and vascular abnormalities but can also be explained by lower diastolic blood pressure. A certain physiological explanation for the observed lower diastolic blood pressure in this study remains obscure. The left ventricle internal diameter (only end-diastolic) showed a small difference between both groups, all values were within the normal range, but significantly higher in PCOS offspring. A potential underlying mechanism is that insulin may act as a growth factor in cardiac myocytes, ${ }^{35,36}$ also androgens could affect fetal cardiac anatomy. ${ }^{37} \mathrm{~A}$ higher ventricle diameter suggests ventricular dilation, a compensatory mechanism to maintain the stroke volume to normal levels in case of decreased ventricular ejection. ${ }^{38}$ In addition, we found lower tissue Doppler imaging of the right wall in systole within the PCOS group.

An important novel finding in the 6- to 8-year old children from mothers with PCOS is the higher carotid IMT in comparison with the reference group. Carotid IMT is a marker of preclinical stages of atherosclerosis and stiffness. ${ }^{39}$ The observed results could indicate that the children of women with PCOS already have subtle arterial dysfunction, which could lead to atherosclerosis in later life. In spite of the limited group size of PCOS children from which blood was withdrawn, there seems to be a metabolic difference: increased concentrations of triglycerides and LDL-cholesterol in the CHOPS group, whereas insulin levels were not significantly different. Previous literature shows that serum insulin levels are significantly higher in prepubertal children of women with PCOS after a 2-hour fasting test with the use of oral glucose, compared to healthy controls. ${ }^{18}$ Dyslipidemia is associated with an increased risk of cardiovascular diseases in later life and could 
already be present during childhood. ${ }^{40}$ These results correspond partially with studies in PCOS offspring at different ages, which showed higher triglycerides, LDL-cholesterol and insulin levels in children from women with PCOS. ${ }^{11,12,18}$ The unfavorable metabolic parameters described are not in line with the lower breast and abdominal circumference in children from women with PCOS regardless of their similar BMI and weight, but significantly lower height. These findings, which contradict previous studies, remain to be confirmed. ${ }^{12,13}$

Early environmental factors influence cardiovascular disease in later life. ${ }^{41}$ Data of a Dutch prospective cohort study additionally suggest that unspontaneous mode of conception might influence offspring's health. ${ }^{42}$ For example, children conceived by ovulation induction (independent from PCOS) exhibit higher glucose, triglycerides, and blood pressures compared to spontaneously conceived children. ${ }^{43}$ Our sensitivity analysis within the PCOS group, however, suggests that there is no significant correlation between the mode of conception and offspring health (data not shown). Furthermore, during pregnancy, mothers of the current PCOS offspring presented with a significantly increased incidence of GDM compared to reference groups (14\% vs $2 \%){ }^{44-46}$ Offspring of diabetic mothers have increased risk of cardiac hypertrophy. ${ }^{47}$ Therefore, we performed a sensitivity analyses showing that GDM was significantly associated with left ventricle internal diameter, end-diastolic $Z$ score (parasternal long axis) in the young group of the WHISTLER cohort (unadjusted and adjusted analyses [age and sex adjusted BMI $Z$ score], $P=.017, P<.001$, respectively). The older CHOPS children exposed to GDM showed higher carotid elastic modulus compared to non-GDM CHOPS offspring $(P<.001)$. In the WHISTLER, children of the older age-group had significantly lower carotid distensiblity when born from a mother with GDM $(P=$ .007). These differences could be caused by a hyperglycemic status of the mothers. ${ }^{47}$ Besides an increased risk of GDM, the risk of hypertensive disorders (PIH and PE) were also increased in the aforementioned 3 meta-analyses. ${ }^{44-46}$ We found no increased incidences of hypertensive disorders in women with PCOS, presumably due to the limited sample size and therefore low power to detect differences. We did not choose to correct for maternal pregnancy complications due to its potential place within the causal chain or mediator function in maternal and offspring health. However, we did perform a sensitivity analyses to generate more insight in the maternal pregnancy complications (PIH, PE, and GDM) as a potential confounder. All outcomes remained significant, except for the children's lipid profiles. The overall MANOVA test on biomarker profiles showed an additionally adjusted $P$ value of .066 .

In contrast of the majority of existing studies, ${ }^{12,18}$ we analyzed both sexes in 1 study since fetuses of both sexes are exposed to similar risk factors during pregnancy in women with PCOS. It remains uncertain whether sex differences in cardiovascular health comparing males and females - so abundantly described in adults ${ }^{48}$ - can already be observed during childhood. In the current study, we noted no overt differences in cardiovascular health comparing male and female children (data not shown).

\section{Limitations}

One of the major limitations was the limited sample size that allows only detection of distinct difference. Furthermore, we cannot fully exclude the possibility that some of the mothers from the reference group were diagnosed with PCOS. This heterogeneity of the reference group should be taken into consideration when interpreting our results. A possible consequence could be that the observed differences comparing PCOS and reference children would be less. A lack of heterogeneity was achieved due to inclusion of mainly Caucasian children, which diminished the generalizability of our study results. Another limitation of this study is that the observers who performed the assessments were not blinded for the PCOS diagnosis of the mother. Unfortunately, we did not have any data on pubertal development. Finally, the age of the included children was mainly determined by the year (2004) we started screening women with PCOS in a standardized fashion. Therefore, the age groups did not fully overlap with the reference group; this was due to the limited number of eligible in the PCOS group.

\section{Strengths}

The extensive screening of children from mothers with PCOS seems quite unique. The broad spectrums of cardiovascular and metabolic risk factors in children are both a strength and weakness since the exact significance of each performed tests in young children remains uncertain. Another strength is that the group of women with PCOS is diagnosed following a standardized extensive phenotyping procedure before conception.

\section{Interpretations}

It remains unknown to what extent current findings of increased metabolic and cardiovascular risk in young children from mothers with PCOS are primarily related to an inherited genetic predisposition, a suboptimal intrauterine environment (hyperglycemia), lifestyle after birth, or a combination of these factors. Intrauterine androgen exposure is a potential pathway for epigenetic modification resulting into an increased risk of metabolic abnormalities in offspring, as shown in Rhesus monkeys. ${ }^{49,50}$

\section{Conclusion}

In conclusion, the current preliminary study demonstrates that the young children of women previously diagnosed with PCOS present with subtle subclinical abnormalities in relation to cardiovascular and metabolic health. These observations require confirmation in independent large data sets. Caution is warranted in PCOS offspring because observed suboptimal conditions may aggravate with increasing age, resulting in increased chances for type 2 diabetes and cardiovascular diseases in later life. 


\section{Authors' Note}

This study was designed by M.A.W, J.B.E., M.P.H.K., A.M.V.E., G.W.D., C.S.P.M.U., M.J.C.E., C.K.E., F.J.M., and B.C.J.M.F. Data collection was done by M.A.W., J.B.E., F.J.M., and A.M.V.E. Retrieved data were analyzed and interpreted by M.A.W, M.N.G., M.J.C.E., and B.C.J.M.F. The manuscript was drafted by M.A.W, M.N.G., M.J.C.E., and B.C.J.M.F. Critical revision of the article was done by M.A.W., J.B.E., M.N.G., F.J.M., and B.C.J.M.F. Final approval of the version to be published by M.A.W, J.B.E., M.N.G., M.P.H.K., A.M.V.E., G.W.D., C.S.P.M.U., M.J.C.E., C.K.E., F.J.M., and B.C.J.M.F. The authors declare complete independence from funders. The medical ethics committee of the UMCU approved the studies of which data were used in this study. Trials were registered at Clinicaltrials.gov with trial number NCT01767051 and NCT02309047 or at the Dutch Trial Register: with registration number NTR1590. All women signed informed consent for using their preconception baseline data from the extensive standardized screening in the fertility clinic for scientific goals. Informed consent of all parents was obtained before screening the children.

\section{Acknowledgments}

The authors would like to acknowledge Liesbeth van der FeltzMinkema and Lidian Izeboud for their major contribution to the data collection.

\section{Declaration of Conflicting Interests}

The author(s) declared no potential conflicts of interest with respect to the research, authorship, and/or publication of this article.

\section{Funding}

The author(s) disclosed receipt of the following financial support for the research, authorship, and/or publication of this article: This study was funded in part by the Child Health research program of the University Medical Centre Utrecht and Dutch Heart Foundation (grant number 2013T083). The Wheezing Illnesses STudy LEidsche Rijn was funded by The Netherlands Organization for Health Research and Development (ZonMw; grant number 2100.0095) and an unrestricted research grant from GlaxoSmithKline. M.N.G. has received funding from the Dutch Heart Foundation, grant number 2013T083. B.C.J.M.F. has over the most recent 5-year period received fees and grant support from the following companies (in alphabetic order): Actavis, COGI, Dutch Heart Foundation, Euroscreen, Ferring, Finox, Gedeon-Richter, OvaScience, PregLem, Pantharei Bioscience, Roche, Uteron, and Watson laboratories.

\section{Supplemental Material}

Supplementary material for this article is available online.

\section{References}

1. Norman RJ, Dewailly D, Legro RS, Hickey TE. Polycystic ovary syndrome. Lancet. 2007;370(9588):685-697.

2. Fauser BC, Tarlatzis BC, Rebar RW, et al. Consensus on women's health aspects of polycystic ovary syndrome (PCOS): the Amsterdam ESHRE/ASRM-sponsored 3rd PCOS consensus workshop group. Fertil Steril. 2012;97(1):28-38.e25.

3. Rotterdam ESHRE/ASRM-Sponsored PCOS consensus workshop group. Revised 2003 consensus on diagnostic criteria and long-term health risks related to polycystic ovary syndrome (PCOS). Hum Reprod. 2004;19(1):41-47.

4. Vink JM, Sadrzadeh S, Lambalk CB, Boomsma DI. Heritability of polycystic ovary syndrome in a Dutch twin-family study. J Clin Endocrinol Metab. 2006;91(6):2100-2104.

5. Crosignani PG, Nicolosi AE. Polycystic ovarian disease: heritability and heterogeneity. Hum Reprod Update. 2001;7(1):3-7.

6. Kosova G, Urbanek M. Genetics of the polycystic ovary syndrome. Mol Cell Endocrinol. 2013;373(1-2):29-38.

7. Moran LJ, Misso ML, Wild RA, Norman RJ. Impaired glucose tolerance, type 2 diabetes and metabolic syndrome in polycystic ovary syndrome: a systematic review and meta-analysis. Hum Reprod Update. 2010;16(4):347-363.

8. Koster MP, de Wilde MA, Veltman-Verhulst SM, et al. Placental characteristics in women with polycystic ovary syndrome. Hum Reprod. 2015;30(12):2829-2837.

9. Palomba S, de Wilde MA, Falbo A, Koster MP, La Sala GB, Fauser BC. Pregnancy complications in women with polycystic ovary syndrome. Hum Reprod Update. 2015;21(5):575-592.

10. Barker DJ. The intrauterine environment and adult cardiovascular disease. Ciba Found Symp. 1991;156:3-10.

11. Kent SC, Gnatuk CL, Kunselman AR, Demers LM, Lee PA, Legro RS. Hyperandrogenism and hyperinsulinism in children of women with polycystic ovary syndrome: a controlled study. J Clin Endocrinol Metab. 2008;93(5):1662-1669.

12. Recabarren SE, Smith R, Rios R, et al. Metabolic profile in sons of women with polycystic ovary syndrome. J Clin Endocrinol Metab. 2008;93(5):1820-1826.

13. Sir-Petermann T, Codner E, Pérez V, et al. Metabolic and reproductive features before and during puberty in daughters of women with polycystic ovary syndrome. J Clin Endocrinol Metab. 2009; 94(6):1923-1930.

14. Steinberg HO, Chaker H, Leaming R, Johnson A, Brechtel G, Baron AD. Obesity/insulin resistance is associated with endothelial dysfunction. Implications for the syndrome of insulin resistance. J Clin Invest. 1996;97(11):2601-2610.

15. Strong JP, Malcom GT, McMahan CA, et al. Prevalence and extent of atherosclerosis in adolescents and young adults: implications for prevention from the pathobiological determinants of atherosclerosis in youth study. JAMA. 1999;281(8):727-735.

16. Legro RS, Kunselman AR, Stetter CM, et al. Normal pubertal development in daughters of women with PCOS: a controlled study. J Clin Endocrinol Metab. 2017;102(1):122-131.

17. Battaglia C, Mancini F, Cianciosi A, et al. Cardiovascular risk in normal weight, eumenorrheic, nonhirsute daughters of patients with polycystic ovary syndrome: a pilot study. Fertil Steril. 2009;92(1):240-249.

18. Sir-Petermann T, Maliqueo M, Codner E, et al. Early metabolic derangements in daughters of women with polycystic ovary syndrome. J Clin Endocrinol Metab. 2007;92(12):4637-4642.

19. Daan NM, Louwers YV, Koster MP, et al. Cardiovascular and metabolic profiles amongst different polycystic ovary syndrome phenotypes: who is really at risk? Fertil Steril. 2014;102(5): 1444-1451.e3.

20. Grobbee DE, Hoes AW, Verheij TJ, Schrijvers AJP, van Ameijden EJ, Numans ME. The Utrecht health project: optimization of 
routine healthcare data for research. Eur J Epidemiol. 2005;20(3): 285-287.

21. Katier N, Uiterwaal CS, de Jong BM, et al. The Wheezing Illnesses Study Leidsche Rijn (WHISTLER): rationale and design. Eur J Epidemiol. 2004;19(9)895-903.

22. Evelein AM, Geerts CC, Visseren FL, et al. The association between breastfeeding and the cardiovascular system in early childhood. Am J Clin Nutr. 2011;93(4):712-718.

23. Dalla Pozza R, Ehringer-Schetitska D, Fritsch P, et al. Intima media thickness measurement in children: a statement from the Association for European Paediatric Cardiology (AEPC) Working Group on Cardiovascular Prevention endorsed by the Association for European Paediatric Cardiology. Atherosclerosis. 2015; 238(2):380-387.

24. Eising JB, van der Ent CK, van der Gugten AC, et al. Life-course of cardio-respiratory associations. Eur J Prev Cardiol. 2015; 22(2):140-149.

25. Lai WW, Geva T, Shirali GS, et al; Task Force of the Pediatric Council of the American Society of Echocardiography; Pediatric Council of the American Society of Echocardiography. Guidelines and standards for performance of a pediatric echocardiogram: a report from the task force of the pediatric council of the American society of echocardiography. J Am Soc Echocardiogr. 2006;19(12):1413-1430.

26. Lopez L, Colan SD, Frommelt PC, et al. Recommendations for quantification methods during the performance of a pediatric echocardiogram: a report from the Pediatric Measurements Writing Group of the American Society of Echocardiography Pediatric and Congenital Heart Disease Council. J Am Soc Echocardiogr. 2010;23(5):465-495.

27. Geerts CC, Evelein AM, Bots ML, van der Ent CK, Grobbee DE, Uiterwaal CS. Body fat distribution and early arterial changes in healthy 5-year-old children. Ann Med. 2012;44(4):350-359.

28. Brands PJ, Hoeks AP, Willigers J, Willekes C, Reneman RS. An integrated system for the non-invasive assessment of vessel wall and hemodynamic properties of large arteries by means of ultrasound. Eur J Ultrasound. 1999;9(3):257-266.

29. Daan NM, Jaspers L, Koster MP, et al. Androgen levels in women with various forms of ovarian dysfunction: associations with cardiometabolic features. Hum Reprod. 2015;30(10):2376-2386.

30. Scherrer U, Rimoldi SF, Rexhaj E, et al. Systemic and pulmonary vascular dysfunction in children conceived by assisted reproductive technologies. Circulation. 2012;125(15):1890-1896.

31. Schulz KF, Grimes DA. Case-control studies: research in reverse. Lancet. 2002;359(9304):431-434.

32. Rothman KJ, Greenland S, Lash TL. Design Strategies to Improve Study Accuracy. Modern Epidemiology. 3rd ed. Philadelphia, PA: Lippincott Williams \& Wilkins; 2008.

33. Pearce N. Analysis of matched case-control studies. BMJ. 2016; 352:i969.

34. Kampmann C, Wiethoff CM, Wenzel A, et al. Normal values of $M$ mode echocardiographic measurements of more than 2000 healthy infants and children in central Europe. Heart. 2000; 83(6):667-672.

35. Hill DJ, Milner RD. Insulin as a growth factor. Pediatr Res. 1985; 19(9):879-886.
36. Ito $\mathrm{H}$, Hiroe $\mathrm{M}$, Hirata $\mathrm{Y}$, et al. Insulin-like growth factor-I induces hypertrophy with enhanced expression of muscle specific genes in cultured rat cardiomyocytes. Circulation. 1993;87(5): 1715-1721.

37. Vyas AK, Hoang V, Padmanabhan V, Gilbreath E, Mietelka KA. Prenatal programming: adverse cardiac programming by gestational testosterone excess. Sci Rep. 2016;6:28335.

38. Kapusta L, Thijssen JM, Groot-Loonen J, Antonius T, Mulder J, Daniels O. Tissue Doppler imaging in detection of myocardial dysfunction in survivors of childhood cancer treated with anthracyclines. Ultrasound Med Biol. 2000;26(7):1099-1108.

39. Raitakari OT, Juonala M, Kahonen M, et al. Cardiovascular risk factors in childhood and carotid artery intima-media thickness in adulthood: the Cardiovascular Risk in Young Finns Study. JAMA. 2003;290(17):2277-2283.

40. Berenson GS, Srinivasan SR, Bao W, Newman WP 3rd, Tracy RE, Wattigney WA. Association between multiple cardiovascular risk factors and atherosclerosis in children and young adults. the Bogalusa Heart Study. N Engl J Med. 1998; 338(23):1650-1656.

41. Roseboom TJ, van der Meulen JH, Osmond C, et al. Coronary heart disease after prenatal exposure to the Dutch famine, 194445. Heart. 2000;84(6):595-598.

42. Ceelen M, van Weissenbruch MM, Vermeiden JP, van Leeuwen FE, Delemarre-van de Waal HA. Cardiometabolic differences in children born after in vitro fertilization: follow-up study. J Clin Endocrinol Metab. 2008;93(5):1682-1688.

43. Pontesilli M, Painter RC, Grooten IJ, et al. Subfertility and assisted reproduction techniques are associated with poorer cardiometabolic profiles in childhood. Reprod Biomed Online. 2015; 30(3):258-267.

44. Boomsma CM, Eijkemans MJ, Hughes EG, Visser GH, Fauser BC, Macklon NS. A meta-analysis of pregnancy outcomes in women with polycystic ovary syndrome. Hum Reprod Update. 2006;12(6):673-683.

45. Kjerulff LE, Sanchez-Ramos L, Duffy D. Pregnancy outcomes in women with polycystic ovary syndrome: a metaanalysis. Am J Obstet Gynecol. 2011;204(6):558.e1-6.

46. Qin JZ, Pang LH, Li MJ, Fan XJ, Huang RD, Chen HY. Obstetric complications in women with polycystic ovary syndrome: a systematic review and meta-analysis. Reprod Biol Endocrinol. 2013; 11:56. doi:10.1186/1477-7827-11-56

47. Elmekkawi SF, Mansour GM, Elsafty MSE, Hassanin AS, Laban M, Elsayed HM. Prediction of fetal hypertrophic cardiomyopathy in diabetic pregnancies compared with postnatal outcome. Clin Med Insights Womens Health. 2015;8:39-43.

48. Mendelsohn ME, Karas RH. Molecular and cellular basis of cardiovascular gender differences. Science. 2005;308(5728): 1583-1587.

49. Bruns CM, Baum ST, Colman RJ, et al. Insulin resistance and impaired insulin secretion in prenatally androgenized male rhesus monkeys. J Clin Endocrinol Metab. 2004;89(12):6218-6223.

50. Abbott DH, Dumesic DA, Eisner JR, Colman RJ, Kemnitz JW. Insights into the development of polycystic ovary syndrome (PCOS) from studies of prenatally androgenized female rhesus monkeys. Trends Endocrinol Metab. 1998;9(2):62-67. 